\title{
A CLOSE ENCOUNTER WITH A SATURN KILOMETRIC RADIATION SOURCE REGION
}

\author{
W. S. Kurth*, D. A. Gurnett*, J. D. Menietti*, R. L. Mutel*, \\ M. G. Kivelson ${ }^{\dagger}$, E. J. Bunce ${ }^{\ddagger}$, S. W. H. Cowley ${ }^{\ddagger}$, D. L. Talboys ${ }^{\ddagger}$,

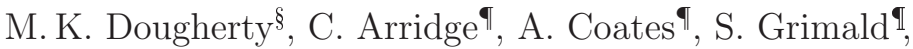 \\ L. Lamy\|, P. Zarkal, B. Cecconi", P. Schippers*, \\ N. Andre**, P. Louarn**, D. Mitchell ${ }^{\dagger \dagger}$, \\ J. S. Leisner*, and M. Morooka ${ }^{\sharp \sharp}$
}

\begin{abstract}
Earth-orbiting satellites have routinely traversed the source regions of auroral kilometric radiation. This radio emission is generated via the cyclotron maser instability very close to the electron cyclotron frequency. While Cassini's orbit has crossed auroral field lines, the radial distance at auroral latitudes is typically too high for the analogous Saturn kilometric radiation source. However, on Oct. 17, 2008, the Radio and Plasma Wave Science instrument detected the kilometric radiation at and just below the electron cyclotron frequency. At this time the spacecraft was at a distance of 5 Saturn radii, at 0.9 hours local time, and on L-shells in the range of 25 to above 30 . Here the magnetic field suggests the corresponding current was directed upward, away from the planet. Low energy electron observations by the Cassini Plasma Spectrometer instrument suggest that growth of the SKR is likely due to an unstable shell-like distribution.
\end{abstract}

\section{Introduction}

It is widely believed that Saturn kilometric radiation (SKR) is produced by the same mechanism as auroral kilometric radiation (AKR) at Earth, that is, via the cyclotron

\footnotetext{
* Department of Physics and Astronomy, University of Iowa, Iowa City, IA 52242, USA

$\dagger$ Institute of Geophysics and Planetary Physics, UCLA, Los Angeles, CA 90095-1567, USA

$\ddagger$ Department of Physics and Astronomy, University of Leicester, Leicester, LE1 7RH, UK

$\S$ Blackett Lab., Imperial College, Prince Consort Road, London SW7 2BZ, UK

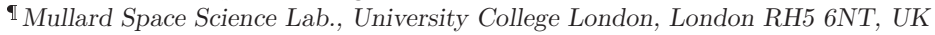

" LESIA, Observatoire de Paris, UMR CNRS 8109, 92195 Meudon, France

${ }^{* *}$ CESR, Université Paul Sabatier, CNRS, 31028 Toulouse 4, France

${ }^{\dagger}$ Applied Physics Lab., Johns Hopkins University, Laurel, MD 20723, USA

${ }_{\ddagger}$ Swedish Institute of Space Physics, SE-751 21 Uppsala, Sweden
} 
maser instability [Wu and Lee, 1979]. Until now, however, there have been no direct observations of the source of SKR with which to verify this or to provide observations with which to compare with the terrestrial source. Here we report on an isolated event in which the SKR frequency drops to and below the local electron cyclotron frequency $\mathrm{f}_{c e}$ and we find evidence for upward directed currents on the source field lines as one would expect. The incomplete electron distributions available from this traversal suggest that the cyclotron maser can, indeed, amplify the waves.

Cassini's orbital tour is shaped to allow observations supporting a wide range of scientific objectives from various latitudes, local times, and radial distances. In late 2008, in order to facilitate the study of Saturn's aurora and associated processes, Cassini was placed into orbits that passed through high latitudes at relatively small radial distances. Such geometries have allowed Cassini to cross auroral field lines at distances of a few to five Saturn radii $\left(\mathrm{R}_{S}=60,268 \mathrm{~km}\right)$. Typically, such distances still leave the spacecraft above the suspected auroral acceleration region and, in particular, above the source region of SKR. However, on orbit 89 on October 17 (day 291), 2008, the Radio and Plasma Wave Science (RPWS) instrument [Gurnett et al., 2004] obtained what appear to be the first in situ observations of an SKR source region. The cyclotron maser instability generates primarily extraordinary mode radiation at approximately $\mathrm{f}_{c e}=28|\mathrm{~B}|$ where $\mathrm{f}_{c e}$ is in $\mathrm{Hz}$ and $|\mathrm{B}|$ is the magnitude of the magnetic field in $\mathrm{nT}$. Hence, one criterion for the SKR source is a region where $\mathrm{f}_{S K R} \approx \mathrm{f}_{c e}$. At the typical minimum auroral field line radial distance of $\sim 5 \mathrm{R}_{S}$ traversed by Cassini, $\mathrm{f}_{c e}$ is $\sim 10 \mathrm{kHz}$. While this is not an unreasonably low frequency for SKR, it is seen less than about $10 \%$ of the time by Cassini [Jackman et al., 2009], typically in conjunction with intensifications associated with a magnetospheric compression caused by a region of high solar wind pressure [Kurth et al., 2005; Jackman et al., 2005].

This paper summarizes the SKR observations along with those of the magnetic field, plasma, and energetic particles relevant to the generation of SKR [Lamy et al., 2010, 2011; Mutel et al., 2010; Bunce et al., 2010; Schippers et al., 2011] and compares them with observations in auroral kilometric radiation source regions at Earth. The Magnetometer (MAG) instrument is described by Dougherty et al. [2004], the Cassini Plasma Spectrometer (CAPS) instrument is described by Young et al. [2004], and the Magnetospheric Imaging Instrument (MIMI) is described by Krimigis et al. [2004].

\section{Observations}

Figure 1 is a frequency-time spectrogram showing the intensity of radio waves as a function of frequency and time for an interval of time on October 17, day 291, 2008, just after periapsis of Cassini's orbit 89. The smoothly varying white trace near $10 \mathrm{kHz}$ is $\mathrm{f}_{c e}$ based on the measured magnetic field. In this time period, SKR dominates the spectrum above $\mathrm{f}_{c e}$ up to nearly $1 \mathrm{MHz}$. This intensification and extension of the SKR spectrum to low frequencies is the expected response from a magnetospheric compression event [Kurth et al., 2005; Jackman et al., 2005]. Notice that between about 8:30 and 9:00 UT the lowfrequency cutoff of the SKR is at or slightly below $\mathrm{f}_{c e}$. The power flux of the SKR near the cutoff is in the range of $10^{-12} \mathrm{Wm}^{-2} \mathrm{~Hz}^{-1}$. The narrowband emission just below $\mathrm{f}_{c e}$ is 
Z-mode radiation associated with narrowband electromagnetic emissions [Ye et al., 2009; Wang et al., 2010]. The broadband emission at even lower frequencies is auroral hiss [Kopf et al., 2010], a whistler mode emission with an upper frequency limit of the lower of $\mathrm{f}_{c e}$ or $\mathrm{f}_{p e}$, the electron plasma frequency. The electron number density $\mathrm{n}_{e}$ is related to $\mathrm{f}_{p e}$ by $\mathrm{f}_{p e}=8980 \sqrt{n_{e}}$ where $\mathrm{f}_{p e}$ is in $\mathrm{Hz}$ and $\mathrm{n}_{e}$ is in $\mathrm{cm}^{-3}$. The variable white trace in Figure 1 is the electron plasma frequency based on Langmuir Probe electron densities inferred from an empirical relation between the spacecraft potential cross-calibrated with electron densities from the CAPS electron spectrometer [Morooka et al., 2009]. During the interval in question the plasma frequency is typically about $500 \mathrm{~Hz}$, hence, $\mathrm{n}_{e} \approx 0.003$ $\mathrm{cm}^{-3}$. The ratio $\mathrm{f}_{p e} / \mathrm{f}_{c e}$ is low, $\sim 0.06$, hence, this ratio is consistent with those required for the cyclotron maser instability. Contrary to the case at Earth, however, there is no clear evidence of a density cavity. We do not know, on the basis of this one case, whether this constitutes a major difference between radio source regions at Earth and Saturn, or if this may simply be due to these particular observations being at or above the top of the acceleration region

Lamy et al. [2010; 2011] provide detailed observations of the SKR near the source (noted by the white ellipse in Figure 1), including the direction of arrival and, importantly, polarization and beaming angles. While the emission is largely propagating in the extraordinary mode, there is a component of ordinary mode emission, not unlike the case at Earth. Further, measurements of the beaming angle show near-perpendicular beaming in the source evolving to a range of angles centered near $70^{\circ}$ further from the source. The emissions near the cutoff are strongly elliptically polarized.

During the interval when the SKR extends down to $\mathrm{f}_{c e}$, Cassini is at a radial distance ranging from about 4.9 to $5.1 \mathrm{R}_{S}$, near $1 \mathrm{hr}$ local time, and at a latitude near $65^{\circ}$ south. The dipole $\mathrm{L}$ shell ranges from about 25 to above 30 during this time, corresponding to an invariant latitude range of 78 to $80^{\circ}$. The inset in Figure 1 shows a cylindrical projection of the source location. It is interesting to note that this location is on the nightside of Saturn. Voyager observations were consistent with a primary SKR source centered in the late local morning, but Farrell et al. [2005], Cecconi et al. [2009], and Lamy et al. [2008; 2009] have used Cassini data to show that SKR sources can be found at any local time.

Figure 2 combines CAPS electron and magnetometer observations with the SKR measurements. A CAPS energy-time spectrogram from the Electron Spectrometer (ELS) portion of the instrument is shown in panel (a). The $\mathrm{r}, \theta$, and $\phi$ components of the magnetic field are shown in panels (b), (c), and (d) after subtracting a model field [Dougherty et al., 2005]. Panel (e) provides an estimate of the Pedersen current per radian based on the $\mathrm{B}_{\phi}$ component. The variations in the $\phi$ component can be related to the passage of the spacecraft from a magnetic field configuration in which the field lines lead in azimuth the configuration of a rigidly co-rotating field to one in which the field line lags, or subcorotates as this component switches sign from negative to positive. The sign of the slope of the current yields the direction of the current. Bunce et al. [2010] report that during this passage there is a central downward-going current flanked on either side by regions of upward current. During the interval between 08:00 and 09:00 UT - when the SKR source is traversed - this current is upward, consistent with downward going electrons as one would expect in an SKR source region [Bunce et al., 2010]. There is structure in both the 


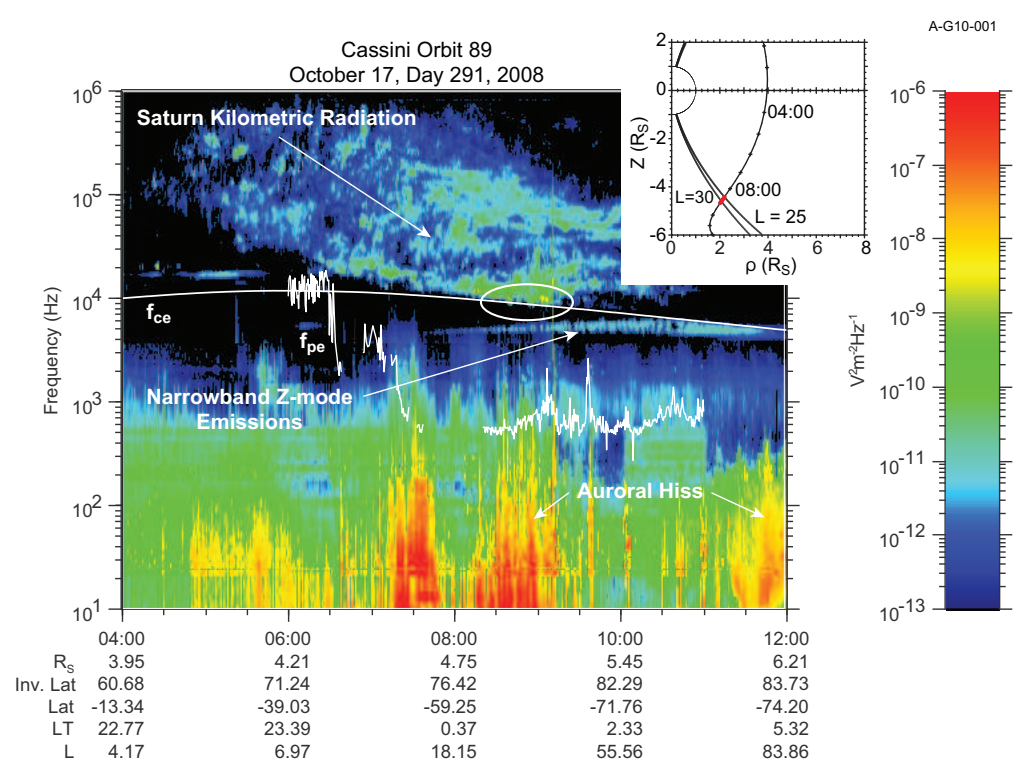

Figure 1: A spectrogram showing the intensity of radio and plasma waves as a function of frequency and time using the color bar on the right. During this interval the SKR intensifies and expands to lower frequencies, reaching the electron cyclotron frequency (smooth white line) in the time interval between $\sim$ 08:30 and 09:00. Just below $f_{c e}$ is an example of narrowband Z-mode emission, and at still lower frequencies is auroral hiss which propagates in the whistler mode. The inset shows a cylindrical projection of the SKR source region highlighted in red between $L$ shells of about 25 and 30.

field and the inferred current, perhaps suggesting filamentary structures imposed on the general current system described by Bunce et al. In particular, the two intervals where the SKR low frequency cutoff drops below $\mathrm{f}_{c e}$ coincide with the steepest positive slopes in the current. A more complete analysis of the magnetic field configuration during the source crossing is given by Bunce et al. [2010]. An expanded view of the SKR data is given in panel (f).

At the lowest energies the ELS spectrogram in Figure 2 is dominated by photoelectrons up to a few to several $10 \mathrm{~s}$ of eV. A plasma sheet-like component is seen at energies above a few hundred $\mathrm{eV}$ up to the $\sim 30 \mathrm{keV}$ upper limit of the instrument. The vertical striations in the spectrum are due to the actuator moving the ELS field of view relative to the magnetic field. The energetic electron population disappears by about 09:00, consistent with the passage from the plasma sheet boundary layer into the lobe (or polar cap) and possibly an indication of the boundary between open and closed field lines. An analysis of the MIMI low energy electron data suggests the open/closed field boundary is at about 08:55 UT. This analysis assumes nearly equal fluxes of low energy (few hundred keV) 


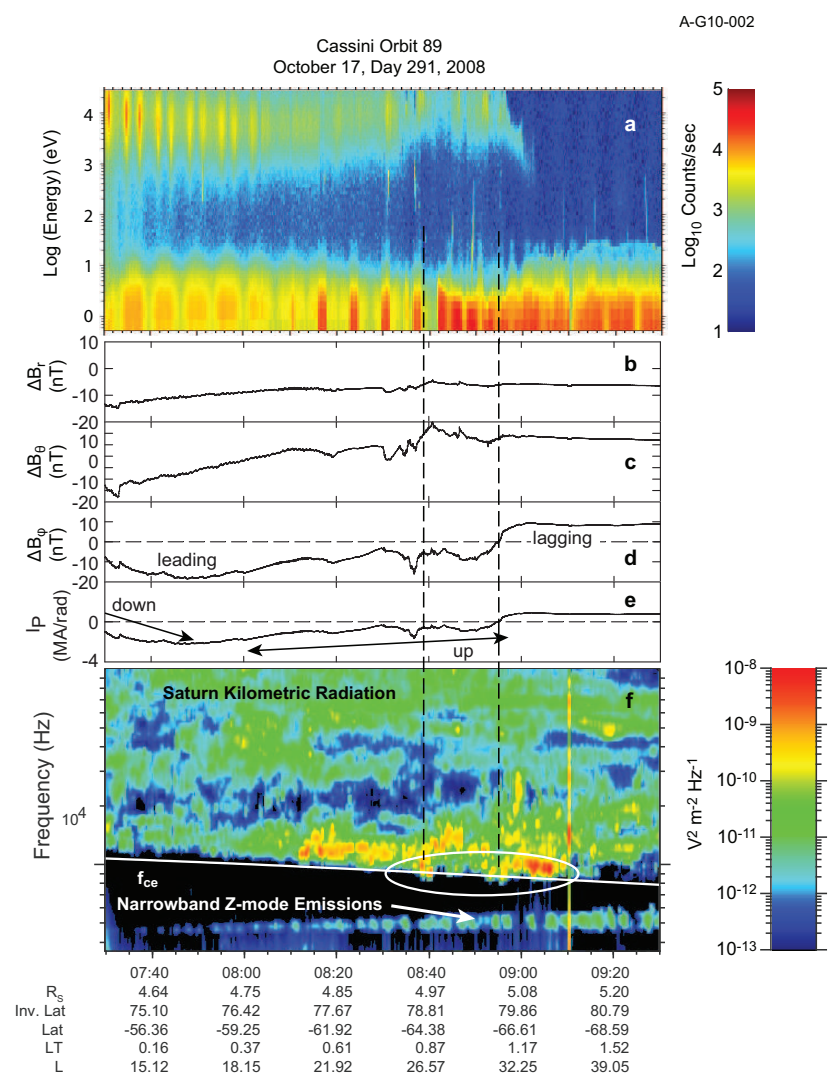

Figure 2: Observations from the CAPS ELS instrument and the Cassini magnetometer are shown along with the RPWS spectrogram of the Saturn kilometric radiation. (a) An ELS energy-time spectrogram averaging data from all anodes. The high fluxes at the lowest energies are due to photoelectrons from the spacecraft. $(b, c, d)$ show the $r, \theta$, and $\phi$ components of the magnetic field with a model field subtracted, respectively. (e) An estimate of the Pedersen current per radian associated with the $\phi$ component of the field assuming the sign of this component can be interpreted as leading or lagging field configurations noted in (d) relative to corotation at low latitudes. A positive slope in this curve is due to an upward-going current. (f) An expanded view of the SKR spectrogram given in Figure 1. The two vertical dashed lines denote when the SKR clearly drops below $f_{c e}$. Note that these times coincide with two of the steepest positive slopes in $B_{\phi}$ and also appear reasonably close to times with brief features in the ELS spectrogram at energies of a few hundred $\mathrm{keV}$.

electrons moving up and down the field line is indicative of closed field lines, but unequal fluxes is an indication of open field lines. In the energy range from below $\sim 100 \mathrm{eV}$ to as high as a few keV, there are occasional very brief bursts of electrons, some of which nearly 
coincide with the times when $\mathrm{f}_{S K R}<\mathrm{f}_{c e}$. Inspection of the orientation of the ELS anodes in which these features are observed indicates that these correspond to upward-going, field-aligned beams.

An analysis of the CAPS electron distribution functions shows that they are nearly featureless except for the brief upgoing beams in the energy range of $\sim$ hundred $\mathrm{eV}$ to a few keV, mentioned above, and evidence of shell-like distributions in the energy range of several to $10 \mathrm{keV}$ [Schippers et al., 2011]. The downgoing electron distribution is largely obscured by parts of the spacecraft. Kopf et al. [2010] show that the low-energy (few hundred $\mathrm{eV}$ ) upgoing beam has a strong positive slope in $\mathrm{v}_{\|}$(velocity parallel to $\mathbf{B}$ ) and can drive the auroral hiss observed at lower frequencies. The high resolution (2-s) ELS data show momentary shell-like distributions [Schippers et al., 2011]. The tail of the ELS electron distributions have a smoothed or plateau-like shape which may have resulted from the relaxation of an initially unstable bump-on-tail distribution. Winglee and Pritchett [1986] suggest that unstable electron distributions can relax on time scales that are short compared to the temporal resolution of the particle detector, hence, the detection of unstable distributions at the higher time resolution as opposed to the longer ones is to be expected. Mutel et al. [2010] have modeled these shells with a Dory-Guest-Harris distribution and found a positive slope in $\mathrm{v}_{\perp}$ (velocity perpendicular to $\mathbf{B}$ ) that can achieve sufficient growth to account for the observed SKR intensities.

MIMI energetic particle observations obtained during this event [Mitchell et al., 2009a] show evidence of ionospheric ions moving upward at the two times when $\mathrm{f}_{S K R}<\mathrm{f}_{c e}$. These are shown in Figure 3. The MIMI INCA instrument was operating in a mode that detects ions and shows intense field-aligned upgoing proton beams in the range of 24 to $90 \mathrm{keV}$ at about 08:37-08:40 and 08:52-08:55. Furthermore, as shown in Figure 5 of Mitchell et al. [2009a], the MIMI CHEMS instrument found that these were exclusively light ions including $\mathrm{H}_{3}^{+}$, consistent with Saturn's ionospheric composition. In the previous hour the ion composition included copious amounts of $\mathrm{O}^{+}$and almost no $\mathrm{H}_{3}^{+}$, consistent with a magnetospheric composition. The ion beams in Figure 3 are consistent with the magnetometer indication of an upward directed current as one would expect for an SKR source region. The intermittent upward-going electron beams mentioned above would seem to imply a downward current, if taken in isolation; however, it is necessary to take into account complete electron and ion distributions (which are not available for this event) before assuming the direction of current based on any particular element of these distributions.

\section{Discussion}

The event described here is the first time Cassini has encountered SKR emissions at or below $\mathrm{f}_{c e}$, even though the spacecraft traced similar geometries several tens of times. It is reasonable that Cassini has only rarely crossed the SKR source region, however. Given that Cassini does not cross auroral field lines much below $5 \mathrm{R}_{S}$, it is necessary that the SKR extend down to $\sim 10 \mathrm{kHz}$ to actually cross the source. The SKR low frequency extensions associated with compression events occur only a couple times per month. Further, these extensions typically last much less than a Saturn rotation and the bright source regions 


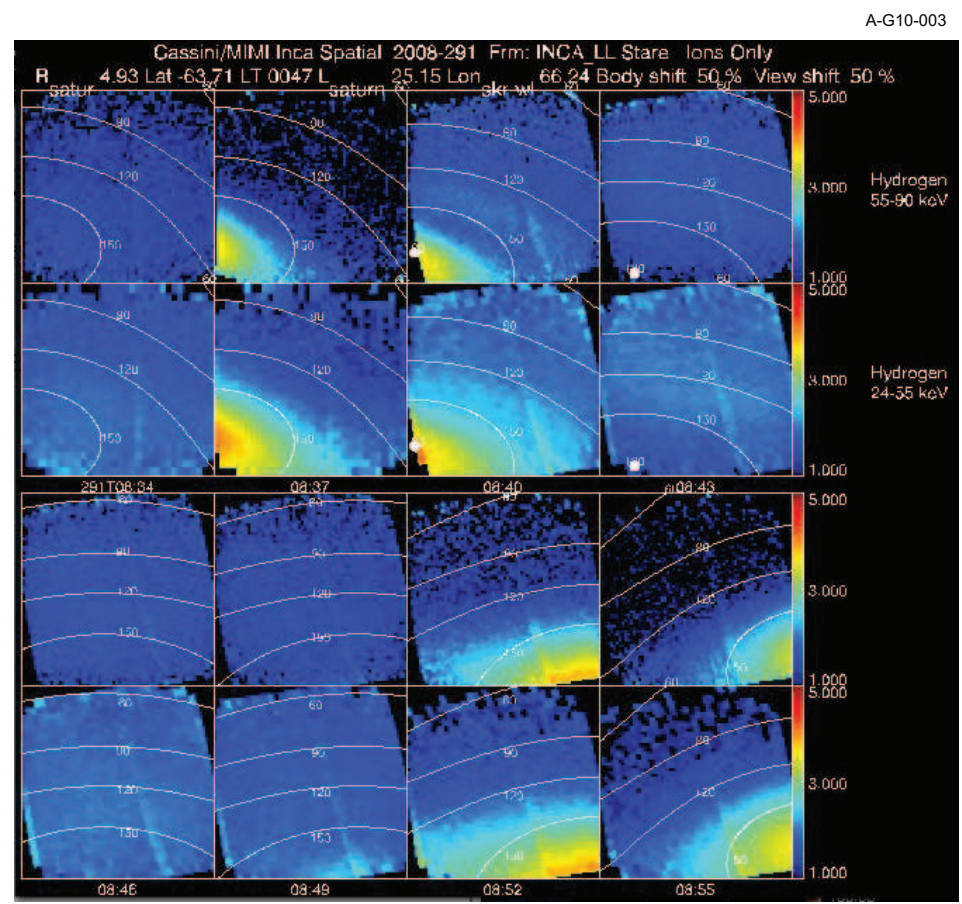

Figure 3: Evidence of field-aligned energetic (50-80) $\mathrm{keV} \mathrm{H}^{+}$observed by the MIMI instrument at the two times when the SKR spectrum extends below the local electron cyclotron frequency. Plotted are the intensities of ions as a function of pitch angle with contours of constant pitch angle superimposed. The beams are tightly confined to pitch angles near 180 degrees, corresponding to the upgoing direction. From Mitchell et al. [2009a].

are limited in longitude. Hence, it is unlikely for Cassini to cross auroral field lines at the right longitude during one of the low frequency extensions.

The local time of this source region is of interest, even though it is not in the late morning sector where the most intense SKR is generated. Mitchell et al. [2009b] have noted concurrent intensifications of SKR, UV auroral emissions, and ENA brightenings in the midnight-to-dawn local time quadrant. That this event is an intensification and lowfrequency extension in the SKR spectrum strongly suggests a temporal initiation of the sort identified by Mitchell et al. Further, the high latitude of this emission, above the latitude for the quiet time auroral oval, is consistent with the poleward extension of auroral activity observed for other magnetospheric compression events [Clarke et al., 2005], although there are no concurrent optical auroral images available during this particular event. Cowley et al. [2005] and Bunce et al. [2005] have suggested that in magnetospheric compression events the tail reconnection rate is considerably larger than the dayside rate and that this reconnection is consistent with the collapse of the magnetotail, the resulting injection of hot plasma from the tail, and the contraction of the polar cap accompanied 
by the poleward expansion of the dawnside aurora.

While the day 291 event meets the necessary condition of the SKR low frequency cutoff extending to and even below $\mathrm{f}_{c e}$, the available electron measurements are challenging for the purposes of providing clear evidence of a distribution function that can drive the cyclotron maser instability [Mutel et al., 2010]. Given that Earth-orbiting spacecraft such as Viking and FAST crossed the auroral acceleration region hundreds or even thousands of times at considerably lower altitudes relative to the auroral acceleration region than in this case, it is perhaps revealing that distributions which are clearly unstable to the cyclotron maser are only fleetingly observed. The particle velocity distributions measured by Cassini over $\sim 3$ minutes are missing an important range of pitch angles that would include downgoing electrons. The only obvious feature in the distributions is an upgoing electron beam. However, higher temporal resolution data reveal evidence of a shell-like distribution near $10-\mathrm{keV}$ that Mutel et al. [2010] have determined can provide sufficient amplification via the cyclotron maser instability to account for the observed emission intensities. The observed shell-like distribution is commonly associated with the auroral kilometric radiation source [c.f. Fung and Viñas, 1994; Louarn et al, 1990; Ergun et al., 2000]. The details of the observed SKR for this event provided by Lamy et al. [2010] strongly suggest that Cassini passed through the source region of the emissions. The arguments are that the distribution of source locations broadened dramatically at the time of the putative source crossing, the spectrum of the SKR is enhanced, and it clearly dropped below the non-relativistic electron cyclotron frequency.

Hence, we have a situation where Cassini has transited L shells that are producing SKR, near the top of the source region corresponding to the bottom of the SKR spectrum. There are momentary measurements of shell-like features in the electron distribution function that appear to provide amplification. In many ways, then, the SKR source region is reminiscent of the AKR source region at Earth.

Acknowledgments. The research at The University of Iowa is supported by NASA through Contract 1356500 with the Jet Propulsion Laboratory.

\section{References}

Bunce, E. J., S. W. H. Cowley, D. M. Wright, A. J. Coates, M. K. Dougherty, N. Krupp, W.S. Kurth, and A.M. Rymer, In situ observations of a solar wind compressioninduced hot plasma injection event in Saturn's tail, Geophys. Res. Lett., 32, L20S01, doi:10.1029/2005GL022888, 2005.

Bunce, E. J., S. W.H. Cowley, D. L. Talboys, M. K. Dougherty, L. Lamy, W.S. Kurth, P. Schippers, B. Cecconi, P. Zarka, C.S. Arridge, and A. J. Coates, Extraordinary field-aligned current signatures in Saturn's high-latitude magnetosphere: Analysis of Cassini data during revolution 89, J. Geophys. Res., 115, A10238, doi:10.1029/ 2010JA015612, 2010.

Cecconi, B., L. Lamy, P. Zarka, R. Prangé, W. S. Kurth, and P. Louarn, Goniopolarimetric study of the rev 29 perikrone using the Cassini radio and plasma wave high-frequency 
radio receiver, J. Geophys. Res., 114, A03215, doi:10.1029/2008JA013830, 2009.

Clarke, J. T., J.-C. Gérard, D. Grodent, S. Wannawichian, J. Gustin, J. Connerney, F. Crary, M. Dougherty, W. Kurth, S. W. H. Cowley, E. J. Bunce, T. Hill, and J. Kim, Morphological differences between Saturn's ultraviolet aurorae and those of Earth and Jupiter, Nature, 433, 717-719, 2005.

Cowley, S. W.H., S. V. Badman, E. J. Bunce, J.T. Clarke, J.-C. Gérard, D. Grodent, C. M. Jackman, S. E. Milan, and T. K. Yeoman, Reconnection in a rotation-dominated magnetosphere and its relation to Saturn's auroral dynamics, J. Geophys. Res., 110, doi:10.1029/ 2004JA010796, 2005.

Dougherty, M. K., S. Kellock, D. J. Southwood, A. Balogh, E. J. Smith, B. T. Tsurutani, B. Gerlach, K.-H. Glassmeier, F. Gleim, C. T. Russell, G. Erdos, F. M. Neubauer, and S. W. H. Cowley, The Cassini magnetic field investigation, Space Sci. Rev., 114, 331-383, 2004.

Dougherty, M.K., et al. (17 co-authors), Cassini Magnetometer observations during Saturn orbit insertion, Science, 307, 1266-1270, doi:10.1126/science.1106098, 2005.

Ergun, R. E., C. W. Carlson, J. P. McFadden, G. T. Delory, R. J. Strangeway, and P. L. Pritchett, Electron-cyclotron maser driven by charge-particle acceleration from magnetic field-aligned electric fields, Astrophys. J., 538, 456-466, doi:10.1086/309094, 2000.

Farrell, W. M., M.D. Desch, M. L. Kaiser, A. Lecacheux, W. S. Kurth, D. A. Gurnett, B. Cecconi, and P. Zarka, A Nightside source of Saturn's kilometric radiation: Evidence for an inner magnetosphere energy driver, Geophys. Res. Lett., 32, L18107, doi:10.1029/2005GL023449, 2005.

Fung, S. F, and A.F. Viñas, Excitation of high-frequency electromagnetic waves by energetic electrons with a loss cone distribution in a field-aligned potential drop, $J$. Geophys. Res., 99, 8671-8686, 1994.

Gurnett, D. A., et al. (29 co-authors), The Cassini radio and plasma wave investigation, Space Sci. Rev., 114, 395-463, 2004.

Jackman, C. M., N. Achilleos, E. J. Bunce, B. Cecconi, J. T. Clare, S. W. H. Cowley, W.S. Kurth, and P. Zarka, Interplanetary conditions and magnetospheric dynamics during the Cassini orbit insertion fly-through of Saturn's magnetosphere, J. Geophys. Res., 110, A10212, doi:10.1029/2005JA011054, 2005.

Jackman, C. M., L. Lamy, M. P. Freeman, P. Zarka, B. Cecconi, W. S. Kurth, S. W. H. Cowley, and M. K. Dougherty, On the character and distribution of lower-frequency radio emissions at Saturn and their relationship to substorm-like events, J. Geophys. Res., 114, A08211, doi:10.1029/2008JA013997, 2009.

Kopf, A., D. A. Gurnett, J. D. Menietti, P. Schippers, C. S. Arridge, G. B. Hospodarsky, W.S. Kurth, S. Grimald, N. André, A. J. Coates, and M. K. Dougherty, Electron beams as the source of whistler-mode auroral hiss at Saturn, Geophys. Res. Lett., 37, L09102, doi:10.1029/2010GL042980, 2010. 
Krimigis, S. M., et al. (24 co-authors), Magnetospheric imaging instrument (MIMI) on the Cassini mission to Saturn/Titan, Space Sci. Rev., 114, 233-329, 2004.

Kurth, W. S., D. A. Gurnett, J. T. Clarke, P. Zarka, M. D. Desch, M. L. Kaiser, B. Cecconi, A. Lecacheux, W. M. Farrell, P. Galopeau, J.-C. Gérard, D. Grodent, R. Prangé, M. K. Dougherty, and F. J. Crary, An Earth-like correspondence between Saturn's auroral features and radio emission, Nature, 433, 722-725, doi:10.1038/nature03334, 2005 .

Lamy, L., P. Zarka, B. Cecconi, R. Prangé, W.S. Kurth, and D. A. Gurnett, Saturn kilometric radiation: Average and statistical properties, J. Geophys. Res., 113, A07201, doi:10.1029/2007JA012900, 2008.

Lamy, L., B. Cecconi, R. Prangé, P. Zarka, J. D. Nichols, and J. T. Clarke, An auroral oval at the footprint of Saturn's kilometric radio sources, colocated with the UV aurorae, J. Geophys. Res., 114, A10212, doi:10.1029/ 2009JA014401, 2009.

Lamy, L., P. Schippers, P. Zarka, B. Cecconi, C. S. Arridge, M. K. Dougherty, P. Louarn, N. André, W.S. Kurth, R. L. Mutel, D. A. Gurnett, and A. J. Coates, Properties of Saturn kilometric radiation measured within its source region, Geophys. Res. Lett., 37, L12104, doi:10.1029/2010GL043415, 2010.

Lamy, L, B. Cecconi, P. Zarka, P. Canu, P. Schippers, W. S. Kurth, R. L. Mutel, D. A. Gurnett, D. Menietti, and P. Louarn, Emission and propagation of Saturn kilometric radiation: Magneto-ionic modes, beaming pattern, and polarization state, J. Geophys. Res., 116, A4, A04212, 2011.

Louarn, P., A. Roux, H. de Feraudy, D. Le Queau, and M. Andre, Trapped electrons as a free energy source for auroral kilometric radiation, J. Geophys. Res., 95, 5983-5995, doi:10.1029/JA095iA05p05983, 1990.

Mitchell, D. G., W. S. Kurth, G. B. Hospodarsky, N. Krupp, J. Saur, B. H. Mauk, J. F. Carbary, S. M. Krimigis, M. K. Dougherty, and D. C. Hamilton, Ion conics and electron beams associated with auroral processes on Saturn, J. Geophys. Res., 114, A02212, doi:10.1029/2008JA013621, 2009a.

Mitchell, D. G., S. M. Krimigis, C. Paranicas, P. C. Brandt, J. F. Carbary, E. C. Roelof, W.S. Kurth, D. A. Gurnett, J. T. Clarke, J. D. Nichols, J.-C. Gérard, D. C. Grodent, M. K. Dougherty, and W.R. Pryor, Recurrent energization of plasma in the midnight-to-dawn quadrant of Saturn's magnetosphere, and its relationship to auroral UV and radio emissions, Planet. Space Sci., 57, 1732-1742, doi:10.1016/j.pss. 2009.04.002, 2009b.

Morooka, M. W., R. Modolo, J.-E. Wahlund, M. André, A. I. Eriksson, A. M. Persoon, D. A. Gurnett, W. S. Kurth, A. J. Coates, G. R. Lewis, K. K. Khurana, and M. Dougherty, The electron density of Saturn's magnetosphere, Ann. Geophys., 27, 29712991, 2009.

Mutel, R. L., J. D. Menietti, D. A. Gurnett, W. Kurth, P. Schippers, C. Lynch, L. Lamy, C. Arridge, and B. Cecconi, CMI growth rates for Saturnian kilometric radiation, Geophys. Res. Lett., 37, L19105, doi:10.1029/2010GL044940, 2010. 
Schippers, P., C. S. Arridge, J. D. Menietti, D. A. Gurnett, L. Lamy, B. Cecconi, D. G. Mitchell, N. André, W. S. Kurth, S. Grimald, M. K. Dougherty, A. J. Coates, N. Krupp, and D. T. Young, Auroral electron distributions within and close to the Saturn kilometric radiation source region, J. Geophys. Res., 116, A5, A05203, 2011.

Wang, Z., D. A. Gurnett, G. Fischer, S.-Y. Ye, W. S. Kurth, D. G. Mitchell, J. S. Leisner, and C. T. Russell, Cassini observations of narrowband radio emissions in Saturn's magnetosphere, J. Geophys. Res., 115, A06213, doi:10.1029/2009JA014847, 2010.

Winglee, R. M., and P.L. Pritchett, The generation of low-frequency electrostatic waves in association with auroral kilometric radiation, J. Geophys. Res., 91, 13531-13541, 1986.

Wu, C.S., and L.C. Lee, A theory of the terrestrial kilometric radiation, Astrophys. J., 230, 621-626, doi:10.1086/157120, 1979.

Ye, S.-Y., D. A. Gurnett, G. Fischer, B. Cecconi, J. D. Menietti, W. S. Kurth, Z. Wang, G. B. Hospodarsky, P. Zarka, and A. Lecacheux, Source locations of narrowband radio emissions detected at Saturn, J. Geophys. Res., 114, A06219, doi:10.1029/ 2008JA013855, 2009.

Young, D. T., et al. (57 co-authors), Cassini plasma spectrometer investigation, Space Sci. Rev., 114, 1-112, 2004. 
